\title{
The sexual agglutinins in Chlamydomonas eugametos are sulphated glycoproteins
}

\author{
Marinus Versluis, * Frans M. Klis, Piet van Egmond and Herman van den Ende \\ Department of Molecular Cell Biology, University of Amsterdam, Kruislaan 318, 1098 SM Amsterdam, \\ The Netherlands
}

(Received 13 July 1992; revised 12 November 1992; accepted 23 November 1992)

\begin{abstract}
Sexual adhesion in the green alga Chlamydomonas eugametos is mediated by hydroxyproline-containing glycoproteins (agglutinins). The agglutinins were metabolically labelled with $\left[{ }^{35}\right.$ S]sulphate. The sulphur-containing amino acids methionine and cysteine were not detectably labelled; little or no sulphated tyrosine was detected. This indicated that the radioactive label had been largely or exclusively incorporated into sulphated sugar residues. Acid hydrolysis released essentially all radioactivity as free sulphate. Short periods of hydrolysis released sulphated oligosaccharides. $\beta$-Elimination released about $20-25 \%$ of the sulphated saccharides, indicating that these are $O$-linked to serine or threonine or both. The remaining sulphated sugar chains are presumably $O$-linked to hydroxyproline. It is proposed that the sulphated sugars of the agglutinins play a role in sexual adhesion.
\end{abstract}

\section{Introduction}

In the biflagellate, unicellular green alga Chlamydomonas eugametos, cell fusion is initiated by adhesion of the flagella between gametes of opposite mating type. This process represents an excellent model system for studying cell-cell recognition (van den Ende et al., 1990). In both mating types, designated $m t^{+}$and $m t^{-}$, hydroxyprolinecontaining glycoproteins, termed agglutinins, are involved in flagellar adhesion (Musgrave et al., 1981; Klis et al., 1985; Samson et al., 1987; Versluis et al., 1992). Both $m t^{+}$and $m t^{-}$agglutinins are long, fibrous molecules (Crabbendam et al., 1986) with estimated molecular masses of 1200 and $1300 \mathrm{kDa}$, respectively (Samson et al., 1987), and they are responsible for the specificity of sexual adhesion (van den Ende et al., 1990).

In many biological processes, including sexual interaction and adhesion, sulphated polysaccharides play an important role. For example, bindin, an adhesive protein from sea urchin sperm, specificially binds sulphated fucan polysaccharides on the surface of the eggs (DeAngelis \& Glabe, 1990). It has also been

*Author for correspondence. Tel. (31) 20 5257848; fax (31) 20 5257715 .

\footnotetext{
Abbreviations: CHAPS, 3-[(3-cholamidopropyl)-dimethylammonio]-1-propanesulphonate; $m t^{-} / m t^{+}$, mating type minus/plus; HP gel filtration, high-performance gel filtration.
}

suggested that adhesive proteins from ram and boar sperm interact with sulphated polysaccharides of the egg (Williams \& Jones, 1990; Jonáková et al., 1991). Finally, fertilization is blocked by the sulphated heteropolysaccharide fucoidan in widely diverging species, suggesting that sulphated polysaccharides mediate this process (Jonáková et al., 1991). Host plant specificity in the Rhizobium-legume symbiosis is determined by $6-O$ sulphation of lipo-oligosaccharide signals (Nod factors) (Roche et al., 1991). Sulphated signal molecules of Rhizobium meliloti elicit root hair curling and nodulation on alfalfa but not on common vetch, while on the other hand nonsulphated factors of mutants of this soil bacterium elicit these biological responses on vetch but not on alfalfa. Other adhesive proteins, including fibronectin (Yamada et al., 1980), laminin, thrombospondin and von Willebrand factor (Roberts et al., 1986), selectively bind sulphated polysaccharides, the binding of which is essential for their biological function.

The following observations suggested that sulphated sugars might also play a role in sexual adhesion of $C$. eugametos gametes. Firstly, the agglutination between gametes of opposite mating type is sensitive to relatively low salt concentrations (Köhle et al., 1980; Goodenough, 1986) and, secondly, sugars appear to be essential for the biological activity of both agglutinins (Samson et al., 1987). Finally, sulphated hydroxyproline-containing glycoproteins are known in green algae. They have been found in the cell wall of Chlamydomonas reinhardtii 
(Roberts et al., 1980) as well as in the extracellular matrix of the related genus Volvox (Ertl et al., 1989). Studies were accordingly undertaken to determine whether or not the sexual agglutinins of $C$. eugametos possess sulphated sugars.

\section{Methods}

Materials. ${ }^{35} \mathrm{~S}$ as carrier-free sulphate in aqueous solution (sp. act. $370 \mathrm{MBq} \mathrm{mmol}{ }^{-1}$; $185 \mathrm{MBq}$ ) was purchased from Amersham. Glycopeptidase F (PNGase F, EC 3.2.2.18) was obtained from Boehringer.

Cell cultures and in vivo ${ }^{35} S$-labelling of the sexual agglutinins. Chlamydomonas eugametos strains 5.39.4 (mt $\left.t^{-}\right)$and 17.17.2 $\left(m t^{+}\right)$were cultivated in Petri dishes on agar-containing medium in a $12 \mathrm{~h}$ light/ $12 \mathrm{~h}$ dark regimen (Schuring et al., 1987). Cells were scraped from 2-3-week-old cultures, which had been cooled for $1 \mathrm{~h}$ at $5{ }^{\circ} \mathrm{C}$, and suspended in $5 \mathrm{~mm}$-HEPES/NaOH buffer, pH 7.2, containing $1 \mathrm{~mm}$ $\mathrm{CaCl}_{2}$ and $1 \mathrm{~mm}-\mathrm{MgCl}_{2} \cdot\left[{ }^{35} \mathrm{~S}\right]$ Sulphate $(37 \mathrm{MBq})$ was added to $20 \mathrm{ml}$ cell suspension $\left(3.5 \times 10^{8}\right.$ cells ml $\left.{ }^{-1}\right)$ and the cells were gently shaken at room temperature in a $12 \mathrm{~h}$ light $/ 12 \mathrm{~h}$ dark regimen. When the cells had incorporated $30-50 \%$ of the added radioactivity (after 1 or $2 \mathrm{~d}$ ), they were harvested. ${ }^{35} \mathrm{~S}$-labelled agglutinins were isolated and purified as described below.

Extraction and purification of agglutinins. The $m t^{-}$and $m t^{+}$ agglutinins were extracted as described previously (Musgrave et al., 1981; Klis et al., 1985). The extracts were fractionated on a ZetaPrep disk (DEAE-anion exchanger; CUNO Inc.). Elution was carried out stepwise with increasing $\mathrm{NaCl}$ concentrations $(50,150,250$, and $500 \mathrm{~mm}$, and $1 \mathrm{M}$ ) in $10 \mathrm{~mm}$-Tris $/ \mathrm{HCl}, \mathrm{pH} \mathrm{7.4}$. Agglutinin-containing fractions, as detected by a semi-quantitative assay (Musgrave et al., 1981), were collected and concentrated with a Centricon-30 microconcentrator (Amicon). The concentrate was applied onto a Bio-Gel TSK-60XL $(7.5 \mathrm{~mm} \times 300 \mathrm{~mm}$; Bio-Rad) gel filtration column. Agglutinin-containing fractions were collected and run again. The column was eluted with $10 \mathrm{~mm}$-sodium phosphate, $100 \mathrm{~mm}$-sodium sulphate at pH 7.4 (Samson et al., 1987). Protein concentrations were calculated from the absorption at $206 \mathrm{~nm}$ (Scopes, 1982).

High-performance gel filtration of hydrolysed ${ }^{35} \mathrm{~S}$-labelled agglutinins. Desulphation of metabolically labelled agglutinin was performed essentially according to Freeze \& Wolgast (1986). Labelled agglutinin was hydrolysed in $0.25 \mathrm{M}-\mathrm{HCl}$ at $100^{\circ} \mathrm{C}$ for different time periods. Samples were cooled and neutralized with $\mathrm{NaOH}$ or $\mathrm{NaHCO}_{3}$. Acid hydrolysates were analysed by HP gel filtration using the following columns connected in series: a Bio-Gel TSK guard column (7.5 $\mathrm{mm} \times 75 \mathrm{~mm})$, a Bio-Gel TSK-10 column $(7.5 \mathrm{~mm} \times 300 \mathrm{~mm}$; BioRad), and a self-packed Bio-Gel P-2 column $(7.5 \mathrm{~mm} \times 600 \mathrm{~mm})$, which separates salts from amino acids and monosaccharides. The columns were eluted with $10 \mathrm{~mm}$-sodium phosphate, $100 \mathrm{~mm}$-sodium sulphate at

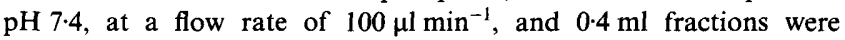
collected. The ${ }^{35} \mathrm{~S}$ activity in each fraction was determined. The column was calibrated with a partial dextran hydrolysate (Yamashita et al., 1982). Carbohydrates were measured by the phenol/sulphuric acid method (Dubois et al., 1956).

$\beta$-Elimination of ${ }^{35} \mathrm{~S}$-labelled agglutinins. Purified, ${ }^{35} \mathrm{~S}$-labelled agglutinin was incubated in $0.05 \mathrm{M}-\mathrm{NaOH}$ and $1 \mathrm{M}-\mathrm{NaBH}_{4}$ for $6 \mathrm{~h}$ at $45^{\circ} \mathrm{C}$ (Lens et al., 1983). The reaction mixture was cooled, titrated with $4 \mathrm{M}-$ acetic acid to $\mathrm{pH} 4 \cdot 5$, and then neutralized with $\mathrm{NaOH}$ and concentrated in a SpeedVac concentrator. The samples were analysed by gel filtration using the HPLC system as described above for the acid hydrolysates or fractionated into high and low molecular mass material by gel filtration on a Sephadex G-25 column $(15 \mathrm{~mm} \times 50 \mathrm{~mm})$.

Sugar and enzyme analysis. Agglutinins were hydrolysed in $0.25 \mathrm{M}$ $\mathrm{HCl}$ for $15 \mathrm{~min}$ at $100^{\circ} \mathrm{C}$. The hydrolysates were applied onto a BioGel P-6 desalting column (cut-off $6000 \mathrm{Da}$ ). High and low molecular mass materials were separately analysed for sugars by gas-liquid chromatography (Gerwig et al., 1984). Glycopeptidase-F (PNG-ase F) incubations were performed in $20 \mathrm{~mm}$-sodium phosphate, $10 \mathrm{mM}$ EDTA, $10 \mathrm{~mm}-\beta$-mercaptoethanol, $0.5 \%$ (w/v) CHAPS, $\mathrm{pH} 7 \cdot 0$, at $37^{\circ} \mathrm{C}$ for $24 \mathrm{~h}$. Samples were heated for $2-3 \mathrm{~min}$ at $100^{\circ} \mathrm{C}$ prior to this treatment.

Barium hydroxide hydrolysis of ${ }^{35} S$-labelled agglutinins. To determine whether tyrosine- $O$-sulphate was present, ${ }^{35} \mathrm{~S}$-labelled agglutinin $(1000-$ 1500 d.p.m.) was hydrolysed with barium hydroxide (Huttner, 1984). After neutralization with sulphuric acid and sedimentation of the precipitate by centrifugation at $10000 \mathrm{~g}$ for $10 \mathrm{~min}$, tyrosine- $O$ sulphate, if present, was in the supernatant fraction. The agglutinins were assayed for the presence of tyrosine- $O$-sulphate by measuring the radioactivity in the neutralized supernatant.

\section{Results}

The presence of sulphate groups in the agglutinins was established by metabolically labelling gametes with $\left[{ }^{35}\right.$ S]sulphate, and isolating and purifying the $m t^{+}$and $m t^{-}$ agglutinins. The standard procedure for purifying the sexual agglutinins consisted of anion-exchange chromatography followed by gel filtration. When the agglutinin-containing fractions were subsequently applied to a $2 \cdot 2 \%-20 \%(\mathrm{w} / \mathrm{v})$ SDS-PAGE gradient gel, a single high-molecular-mass glycoprotein was observed (Samson et al., 1987). For the present set of experiments, the agglutinin-containing fractions of the first gel filtration run were isolated and applied again to the same column. A single peak of ${ }^{35} \mathrm{~S}$ activity was observed which coincided with a single absorption peak at $206 \mathrm{~nm}$. The specific activities were $73 \pm 10 \mathrm{~Bq} \mu \mathrm{g}^{-1}$ (mean $\pm \mathrm{SEM} ; n=$

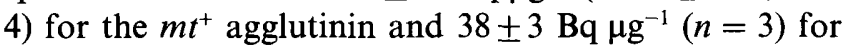
the $m t^{-}$agglutinin, corresponding to at least 238 and 130 sulphur atoms, respectively, per molecule of agglutinin.

There are three known possibilities by which sulphur can occur in glycoproteins. Firstly, it can be incorporated into the sulphur-containing amino acids methionine and cysteine. Secondly, sulphation of sugar residues can occur. Thirdly, sulphate can be $O$-esterified to tyrosine (Huttner, 1984). If present, tyrosine- $O$-sulphate can be detected in the supernatant fraction after $\mathrm{Ba}(\mathrm{OH})_{2}$ hydrolysis and subsequent neutralization with sulphuric acid to co-precipitate released sulphate as its barium salt (Huttner, 1984). However, this treatment released only $4-6 \%$ of the ${ }^{35} \mathrm{~S}$ label of both agglutinins into the supernatant fraction (not shown). It was thus concluded that the agglutinins possessed little or no tyrosine- $O$ sulphate and that about $95 \%$ of the ${ }^{35} \mathrm{~S}$ label had been incorporated otherwise. 


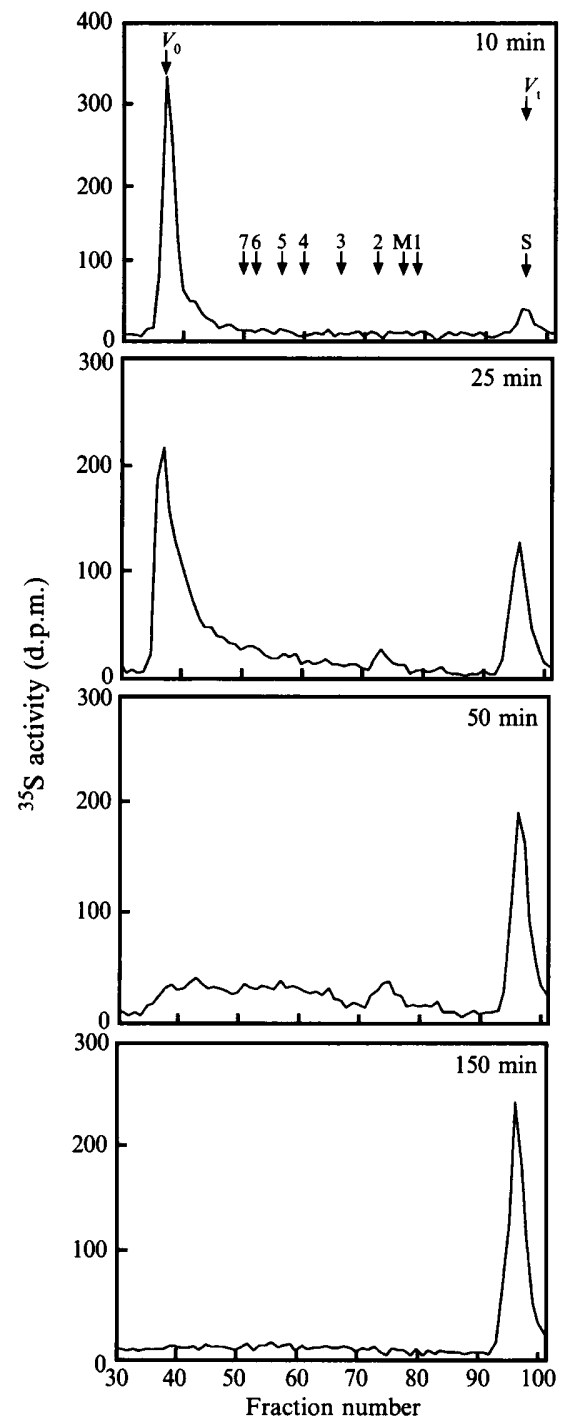

Fig. 1. HP gel filtration analysis of ${ }^{35} \mathrm{~S}$-labelled $m t^{+}$agglutinin after mild acid hydrolysis. Incubations were performed in $0.25 \mathrm{M}-\mathrm{HCl}$ at $100{ }^{\circ} \mathrm{C}$ for different periods of time. The percentages of the total ${ }^{35} \mathrm{~S}$ activity eluting in fractions $40-89$ were as follows: $22 \%$ (hydrolysis for $10 \mathrm{~min}$ ), $43 \%$ ( $25 \mathrm{~min}$ ), $56 \%$ (50 $\mathrm{min}$ ), and $22 \%$ (150 min). Arrows indicate the following elution positions: $V_{0}$, (void volume) - the cut-off was about $2300 \mathrm{Da} ; V_{\mathrm{t}}$, total volume; 1 , glucose; 2 , sucrose; 3 , raffinose; 4 to 7 , dextran hydrolysate, presumably $\mathrm{Glc}_{4}$ to $\mathrm{Glc}_{7} ; \mathrm{M},\left[{ }^{35} \mathrm{~S}\right]$ methionine; and $\left.\mathrm{S},{ }^{35} \mathrm{~S}\right]$ sulphate. The fraction volume was $0.4 \mathrm{ml}$. Similar HP gel filtration profiles were obtained for the $m t^{-}$agglutinin.

In order to discriminate between the other two possibilities, ${ }^{35}$ S-labelled agglutinins were subjected to mild acid hydrolysis for different periods of time. The hydrolysates were analysed by HP gel filtration. Increasing hydrolysis periods resulted in a gradual shift of ${ }^{35} \mathrm{~S}$ activity from void-volume to total-volume fractions, where free sulphate elutes after monosaccharides and single amino acids (Fig. 1). After acid hydrolysis for $150 \mathrm{~min}$, all the ${ }^{35} \mathrm{~S}$ activity eluted as free sulphate in the
Table 1. Composition of the saccharides released from the sexual agglutinins as a percentage of the total amount of each monosaccharide (Klis et al., 1989) after acid hydrolysis for $15 \mathrm{~min}$ in $0.25 \mathrm{M}-\mathrm{HCl}$ at $100^{\circ} \mathrm{C}$.

The means of two separate determinations are presented. The variations in the sugar determinations were less than $10 \%$.

\begin{tabular}{lcc}
\hline \hline & \multicolumn{2}{c}{ Composition (\%) of saccharides released from } \\
\cline { 2 - 3 } Sugar & $m t^{-}$agglutinin & $m t^{+}$agglutinin \\
\hline Ara & 91 & 94 \\
Rha & 60 & 100 \\
Xyl & 81 & 96 \\
Man & 75 & 98 \\
Gal & 89 & 86 \\
Glc & 94 & 96 \\
GlcNAc & 73 & $<1$ \\
\hline \hline
\end{tabular}

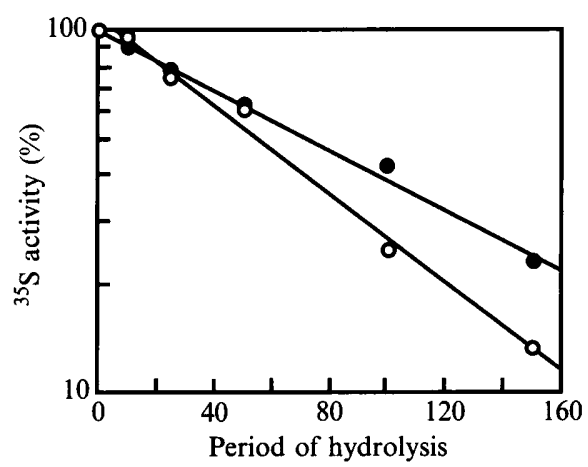

Fig. 2. Determination of half-lives of the release of free $\left[{ }^{35} \mathrm{~S}\right]$ sulphate from labelled agglutinins in $0.25 \mathrm{M}-\mathrm{HCl}$ at $100^{\circ} \mathrm{C}$.,$m t^{+}$agglutinin; $\bigcirc, m t^{-}$agglutinin.

total volume. No radioactivity was found in cysteine or methionine. Thus, the ${ }^{35} \mathrm{~S}$ label in both agglutinins was predominantly or exclusively present as sugar-linked sulphate groups. Depending on the duration of hydrolysis, intermediate compounds $(\leqslant 2300 \mathrm{Da})$ were also found. The percentage of radioactivity in the fractions between the void and total volume (viz. fractions 40-89 in Fig. 1) increased from $22 \%$ after a 10 min hydrolysis to $56 \%$ after a $50 \mathrm{~min}$ hydrolysis. The results for the $m t^{-}$ agglutinin were $19 \%$ and $59 \%$, respectively (not shown). This suggested that both agglutinins had been partially deglycosylated and had released sulphated oligosaccharides. This was supported by gas chromatographic analysis of the released material, which showed that after 15 min all major sugars were, to a large extent, released (Table 1). The $t_{\frac{1}{2}}$ for sulphate release appeared to be $74 \mathrm{~min}$ for the $m t^{+}$agglutinin and $53 \mathrm{~min}$ for the $m t^{-}$ agglutinin (Fig. 2). These half-lives indicate an axial position of the sulphate residues in the sugar rings (Rees, 1963). 


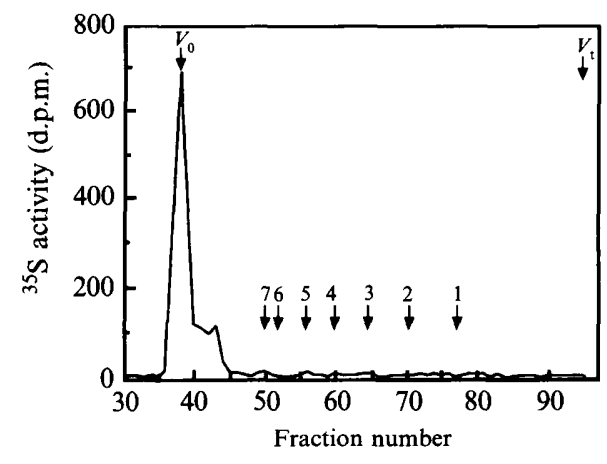

Fig. 3. HP gel filtration analysis of ${ }^{35} \mathrm{~S}$-labelled $\mathrm{mt}^{+}$agglutinin after $\beta$ elimination. The $\beta$-elimination products eluted after the void volume $\left(V_{0}\right)$ and contained $21 \%$ of the total ${ }^{35} \mathrm{~S}$ activity (fractions $40-45$ ). Arrows indicate the following elution positions: $V_{0}$, void volume - the cut-off was about $2300 \mathrm{Da} ; V_{\mathrm{t}}$, total volume; 1 , glucose; 2 , sucrose; 3 , raffinose; 4 to 7, dextran hydrolysate, presumably $\mathrm{Glc}_{4}$ to $\mathrm{Glc}_{7}$. An $\mathrm{HP}$ gel filtration profile representative of four individual experiments is presented. Similar results were obtained for the $m t^{-}$agglutinin.

The $O$-glycosidic bond between sugars and the $\beta$ hydroxyamino acids, serine and threonine, is relatively sensitive to alkaline cleavage by a $\beta$-elimination type reaction (Sharon, 1975). In the presence of the strong reducing agent $\mathrm{NaBH}_{4}$, the released oligosaccharide sidechains can be recovered to determine whether or not they contain ${ }^{35} \mathrm{~S}$ label. $\beta$-Elimination of both agglutinins released $30 \%$ of the sulphated sugars as low-molecularmass material recovered after Sephadex G-25 gel filtration (not shown). After HP gel filtration of the $\beta$ elimination products of the $m t^{+}$agglutinin, approximately $20-25 \%$ of the ${ }^{35} \mathrm{~S}$-label eluted directly after the void volume peak (viz. fractions $40-45$ in Fig. 3). These oligosaccharides were at least nine residues long as determined with a partial dextran hydrolysate for standards. The results of Sephadex G-25 gel filtration and HP gel filtration of the $\beta$-elimination products showed that a considerable proportion of the sulphate groups were attached to sugars $O$-glycosidically linked to serine or threonine or both. The alkali-insensitive part of the ${ }^{35} \mathrm{~S}$ label is presumably exclusively attached to hydroxyproline-linked saccharides, since treatment with glycopeptidase-F, which hydrolyses $N$-glycosidic bonds, did not release any labelled material.

\section{Discussion}

In this report both the $m t^{+}$and $m t^{-}$sexual agglutinins of Chlamydomonas eugametos are shown to be sulphated glycoproteins. Sulphation is a post-translational modification which results in sulphate being linked as an ester to either tyrosine or sugar residues. Our results indicate that in these agglutinins, sulphate is largely or exclusively bound to sugars $O$-linked to either serine and threonine or to hydroxyproline. By $\beta$-elimination it was shown that about a quarter of the ${ }^{35} \mathrm{~S}$ label was present in $O$-linked sugars attached to serine or threonine. The remainder is probably attached to glycosides linked to hydroxyproline.

After mild acid hydrolysis, a number of labelled products were obtained which probably represent sulphated oligosaccharides and free sulphate. The high sensitivity to acid hydrolysis of the sugar side-chains in the sexual agglutinins is in agreement with observations on other hydoxyproline-containing glycoproteins. Firstly, because arabinose is one of the predominant sugars in the sexual agglutinins (Samson et al., 1987; Klis et al., 1989), it seems likely that the sexual agglutinins of $C$. eugametos will contain arabinofuranosyl hydroxyproline linkages as similar hydroxyproline-containing glycoproteins do (Lamport, 1980). Such linkages are very acidlabile: they can be hydrolysed within $1 \mathrm{~h}$ in $0 \cdot 1 \mathrm{M}-\mathrm{HCl}$ at $100^{\circ} \mathrm{C}$ (Lamport, 1980). Secondly, Miller et al. (1972) found that hydroxyproline glycosides isolated from Chlamydomonas cell wall protein could be partially degraded by treatment with $0 \cdot 1 \mathrm{M}$-trifluoroacetic acid at $90{ }^{\circ} \mathrm{C}$ for $30 \mathrm{~min}$.

The half-lives for release of free sulphate of both agglutinins differ from those found for sulphated cell wall glycoproteins in C. reinhardtii (Roberts et al., 1980), implying that sugars in $C$. eugametos agglutinins are esterified at different positions. The occurrence of sulphate groups in both agglutinins probably also explains the low isoelectric points of both the $m t^{+}$ agglutinin (pI 4.3) and the $m t^{-}$agglutinin (pI 3) (Samson et al., 1987).

The fact that sulphated carbohydrate side-chains are often involved in biological recognition and in the binding and interaction of macromolecules raises the question whether or not the sulphated oligosaccharide side-chains in the sexual agglutinins play a role in sexual agglutination. The following observations indicate that they might indeed be involved in this process. Relatively low concentrations of salts can completely and reversibly inhibit sexual adhesion in Chlamydomonas (Köhle et al., 1980; Goodenough, 1986), suggesting that the adhesion reaction is due to ionic binding of positively and negatively charged groups at the recognition site. On the other hand, there is strong evidence that in both sexual agglutinins sugar residues are essential for the biological activity of the agglutinins. For example, both molecules lose their activity after a short treatment with periodate, which specifically attacks sugars (Woodward et al., 1985). It is, therefore tempting to speculate that the sulphated sugars in both agglutinins play a role in sexual adhesion. 
These investigations were supported by the Foundation for Biological Research (BION), which is subsidized by the Netherlands Organization for Scientific Research (NWO).

\section{References}

Crabbendam, K. J., Klis, F. M., Musgrave, A. \& van den Ende, H. (1986). Ultrastructure of the plus and minus mating-type sexual agglutinins of Chlamydomonas eugametos, as visualized by negative staining. Journal of Ultrastructure and Molecular Structure Research 96, 151-159.

DeAngelis, P. L. \& Glabe, C. G. (1990). Specific recognition of sulphate esters by bindin, a sperm adhesion protein from sea urchins. Biochimica et Biophysica Acta 1037, 100-105.

Dubois, M., Gilles, K. A., Hamilton, J. K., Rebers, P. A. \& Smith, F. (1956). Colorimetric method for determination of sugars and related substances. Analytical Chemistry 28, 350-356.

van den Ende, H., Musgrave, A. \& Klis, F. M. (1990). The role of flagella in the sexual reproduction of Chlamydomonas gametes. In Ciliary and Flagellar Membranes, pp. 129-147. Edited by R. A. Bloodgood. London \& New York: Plenum.

Ertl, H., Mengele, R., Wenzl, S., Engel, J. \& Sumper, M. (1989). The extracellular matrix of Volvox carteri: molecular structure of the cellular compartment. Journal of Cell Biology 109, 3493-3501.

FrEeZE, H. H. \& WolgaST, D. (1986). Structural analysis of $N$-linked oligosaccharides from glycoproteins secreted by Dictyostelium discoideum. Journal of Biological Chemistry 261, 127-134.

Gerwig, G. J., Kamerling, J. P., Vliegenthart, J. F. G., Homan, W. L., van EgMOND, P. \& VAN DEN ENDE, H. (1984). Characteristic differences in monosaccharide composition of glycoconjugates from opposite mating types of Chlamydomonas eugametos. Carbohydrate Research 127, 245-251.

GoODENOUGH, U. W. (1986). Experimental analysis of the adhesion reaction between isolated Chlamydomonas flagella. Experimental Cell Research 166, 237-246.

HUTTNER, W. B. (1984). Determination and occurrence of tyrosine- $O$ sulphate in proteins. Methods in Enzymology 107, 200-223.

Jonáková, V., Sanz, L., Calvete, J. J., Henschen, A., Cechova, D. \& TÖPFER-PETERSEN, E. (1991). Isolation and biochemical characterization of a zona pellucida-binding glycoprotein of boar sperm. FEBS Letters 280, 183-186.

Klis, F. M., Samson, M. R., Touw, E., Musgrave, A. \& van den ENDE, H. (1985). Sexual agglutination in the unicellular green alga Chlamydomonas eugametos - identification and properties of the mating type plus agglutination factor. Plant Physiology 79, 740-745.

Klis, F. M., Crabbendam, K., van Egmond, P. \& van den Ende, H. (1989). Ultrastructure and properties of the sexual agglutinins of the biflagellate green alga Chlamydomonas moewusii. Sexual Plant Reproduction 2, 213-218.

KöHLE, D., LaNG, W. \& Kauss, H. (1980). Agglutination and glycosyltransferase activity of isolated gametic flagella from Chlamydomonas reinhardii. Archives of Microbiology 127, 239-243.
LAMPORT, D. T. A. (1980). Structure and function of plant glycoproteins. In The Biochemistry of Plants, vol. 3, pp. 501-541. Edited by J. Preiss. New York: Academic Press.

Lens, P. F., Olofsen, F., van Egmond, P., Musgrave, A. \& van den ENDE, H. (1983). Isolation of an antigenic determinant from flagellar glycoproteins of Chlamydomonas eugametos. Archives of Microbiology 135, 311-314.

Miller, D. H., Lamport, D. T. A. \& Miller, M. (1972). Hydroxyproline heterooligosaccharides in Chlamydomonas. Science 176, 918-920.

Musgrave, A., van Eyk, E., te Welscher, R., Broekman, R., Lens, P., Homan, W. \& VAN DEN ENDE, H. (1981). Sexual agglutination factor from Chlamydomonas eugametos. Planta 153, 362-369.

REES, D. A. (1963). A note on the characterization of carbohydrate sulphates by acid hydrolysis. Biochemical Journal 88, 343-345.

Roberts, D. D., RaO, C. N., Liotta, L. A., Gralnick, H. R. \& GINSBERG, V. (1986). Comparison of the specificities of laminin, thrombospondin, and von Willebrand factor for binding to sulphated glycolipids. Journal of Biological Chemistry 261, 6872-6877.

RoberTs, K., Gay, M. R. \& Hills, G. J. (1980). Cell wall glycoproteins from Chlamydomonas reinhardii are sulphated. Physiologia Plantarum 49, 421-424.

Roche, P., Debellé, F., Maillet, F., Lerouge, P., Faucher, C., TruChet, G., Dénarié, J. \& Promé, J.-C. (1991). Molecular basis of symbiotic host specificity in Rhizobium meliloti: nodH and nodPQ genes encode the sulphation of lipooligosaccharide signals. Cell 67, 1131-1143.

Samson, M. R., Klis, F. M., Homan, W. L., van Egmond, P., Musgrave, A. \& Van den Ende, H. (1987). Composition and properties of the sexual agglutinins of the flagellated green alga Chlamydomonas eugametos. Planta 170, 314-321.

Schuring, F., SmeEnK, J. W., Homan, W. L., Musgrave, A. \& van DEN ENDE, H. (1987). Occurrence of $O$-methylated sugars in surface glycoconjugates in Chlamydomonas eugametos. Planta 170, 322-327.

ScOPEs, R. (1982). Measurement of enzyme activity. In Protein Purification. Principles and Practice, pp. 253-283. Edited by C. R. Cantor. New York: Springer-Verlag.

Sharon, N. (1975). Carbohydrate-peptide linkages. In Complex Carbohydrates. Their Chemistry, biosynthesis, and Functions, pp. 65-83. Reading, Massachusetts: Addison-Wesley.

Versluis, M., Schuring, F., Klis, F. M., VAN EGMOND, P. \& VAN DeN ENDE, H. (1992). Sexual agglutination in Chlamydomonas eugametos is mediated by a single pair of hydroxyproline-rich glycoproteins. FEMS Microbiology Letters 97, 101-105.

Williams, R. M. \& Jones, R. (1990). Specific binding of sulphated polymers to ram sperm proacrosin. FEBS Letters 270, 168-172.

WoOdWARd, M. P., Young, W. W., JR \& BloOdgoOd, R. A. (1985). Detection of monoclonal antibodies specific for carbohydrate epitopes using periodate oxidation. Journal of Immunological Methods 78, 143-153.

Yamada, K. M., Kennedy, D. W., Kimata, K. \& Pratt, R. M. (1980). Characterization of fibronectin interactions with glycosaminoglycans and identification of active proteolytic fragments. Journal of Biological Chemistry 255, 6055-6063.

Yamashita, K., Mizuochi, T. \& Kobata, A. (1982). Analysis of oligosaccharides by gel filtration. Methods in Enzymology 83, $105-126$. 